\title{
Development and in vitro characterisation of a benznidazole liposomal formulation
}

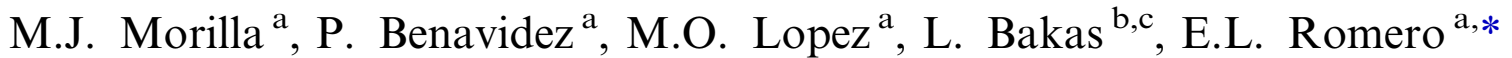 \\ ${ }^{\text {a }}$ Laboratorio de Diseño de Transportadores de Drogas, Universidad Nacional de Quilmes, Roque Saenz Peña 180, Bernal B1876BXD, \\ Buenos Aires, Argentina \\ ${ }^{\mathrm{b}}$ Comisión de Investigaciones Cientificas, Instituto de Investigaciones Bioquímicas de La Plata (INIBIOLP), La Plata, Argentina \\ ${ }^{\mathrm{c}}$ Departamento de Ciencias Biológicas, Facultad de Ciencias Exactas-UNLP, La Plata, Argentina
}

Received 28 February 2002; received in revised form 12 August 2002; accepted 13 August 2002

\begin{abstract}
The purpose of this study was to find a multilamellar liposomal formulation for the antichagasic drug Benznidazole (BNZ). Different lipid matrices and organic solvents for BNZ were tested in order to obtain the liposomes with the highest $\mathrm{g}$ BNZ/100 g total lipid ( $D / T L)$ ratio. The best lipid matrices resulted from hydrogenated phosphatidylcholine from soybean (HSPC): Cholesterol (Chol): distearoyl-phosphatidylglycerol (DSPG) (molar ratio 2:2:1) prepared with BNZ dissolved in DMSO. Drug loading of $2 \mathrm{~g} \mathrm{BNZ/100} \mathrm{g}$ total lipids at a total lipid concentration of 20-30 mM was obtained. Two in vitro assays on the HSPC:Chol:DSPG formulation to predict its in vivo behaviour were performed. In the first experiments, after $60 \mathrm{~min}$ at $1-450$-fold dilution in buffer at $37{ }^{\circ} \mathrm{C}$, the amount of drug associated to liposomes was reduced from 2 to $0.25 \mathrm{~g} \mathrm{BNZ} / 100 \mathrm{~g}$ total lipids at a rate of $65 \%$ (drug lost) $\mathrm{min}^{-1}$ at the first minute followed by $0.4 \%$ (drug lost) $\mathrm{min}^{-1}$ during the next hour. When incubated in plasma at $37{ }^{\circ} \mathrm{C}$, the HSPC:Chol:DSPG formulations bounded a high amount of plasma proteins: $r=2400 \mu \mathrm{g}$ plasma protein per $\mu \mathrm{mol}$ total lipid.
\end{abstract}

(C) 2002 Elsevier Science B.V. All rights reserved.

Keywords: Multilamellar liposomes; Benznidazole

\section{Introduction}

Nowadays, Chagas' disease affects around 20 million people in Central and South America alone. In spite of efforts done to develop new chemoterapeutics or vector control strategies to

\footnotetext{
* Corresponding author. Tel.: +54-11-4365-7100; fax: +5411-4365-7132

E-mail address: elromero@unq.edu.ar (E.L. Romero).
}

defeat Chagas' disease, the Argentinean pharmaceutical market only has BNZ as the solely approved drug available for human treatments (Van Gompel and Vervoort, 1997). When orally administered at the acute phase of the disease, BNZ rapidly and efficiently eliminates the circulating (trypomastigotes forms) parasites. In chronic or reactivation episodes, BNZ efficacy is much lower and chemotherapy must be interrupted due to serious side effects, dealing with 
disturbances associated to the digestive tract such as nausea, vomiting and loss of weight, dermopathy, cutaneous morbiliforms eruptions and polyneuropathy (Coura, 1996).

The transformation of a free drug into its liposomal formulation is a strategy to control and modify its pharmacokinetics and biodistribution. After entrapment, both parameters depend mainly on liposomal physico-chemical properties. Thus, liposomal formulations could surpass the problems of BNZ's low water solubility, instability and free accessibility to all tissues drug. By manufacturing multi or unilamellar, large or small vesicles, and properly choosing the nature of their lipids it is possible to exert a control upon drug delivery by liposomal carriers. There are poor expectations for the BNZ as ideal drug to be loaded in liposomes (for instance, its low hydrosolubility and absence of weak acid-base behaviour difficult both its retention in phospholipid bilayers upon dilution and the employment of an active loading method to obtain a high drug/lipid ratio). Because of this, our main concern was to test the in vitro performance of a series of lipid matrices in order to select that most suitable as liposomal carrier for BNZ. The preparation of a structurally stable liposomal formulation should be the first step towards obtaining a therapeutic approach potentially capable of reducing the toxic side effects related to the high doses of the free drug.

\section{Materials and methods}

\subsection{Materials}

Phosphatidylcholine from fresh egg yolk (EggPC), hydrogenated phosphatidylcholine from soybean (HSPC), distearoyl-phosphatidylglycerol (DSPG), dipalmitoyl-phosphatidylethanolamine (DPPE), Cholesterol (chol), Tris buffer and Nycodenz were from Sigma St. Louis MO, USA. Laurdan was from Molecular Probes, Eugene, USA. Dimethy sulfoxide (DMSO) was from Anedra and Benznidazole (BNZ) a gift from
Roche, Argentina. All reagents were analytical grade.

\subsection{BNZ hydrophobic/hydrophilic balance}

The BNZ hydrophobic/hydrophilic balance was estimated from the partition coefficient $\mathrm{Kp}=$ [drug in liposomes $/ \mathrm{drug}$ in aqueous medium], according to Lissi (Lissi et al., 1990). EggPC multilamellar vesicles were extruded through $200 \mathrm{~nm}$ polycarbonate membranes (using a Avanti Mini-Extruder device) to form unilamellar vesicles of $10 \mathrm{mM}$ total lipids in Tris buffer $20 \mathrm{mM}, \mathrm{pH}$ 7.4. The fluorescent dye Laurdan was added from a methanolic solution, to $3 \mathrm{ml}$ of pre-formed liposomes, in a dye: total lipid ratio of 1:400 (mol: $\mathrm{mol}$ ) and then incubated at room temperature in the dark for $1 \mathrm{~h}$. The fluorescence spectra were recorded on a SLM 4800 Aminco Bowman Spectrofluorometer with a temperature controlled sample holder at $25{ }^{\circ} \mathrm{C}$. Slit width was $8 \mathrm{~nm}$ for both excitation and emission. Upon excitation at $360 \mathrm{~nm}$, the emission spectrum was recorded as a function of successive additions (25-350 nanomols) of BNZ from methanolic solution at $5 \mu \mathrm{mol} \mathrm{ml}{ }^{-1}$. The addition of methanolic solution of BNZ was lower than $2 \%$ of the aqueous phase volume. The parameter $I_{0} / I$ was determined after each BNZ addition.

\subsection{Preparation of BNZ-loaded liposomes}

Multilamellar liposomes were prepared by mixing lipids from $\mathrm{Cl}_{3} \mathrm{CH}$ : $\mathrm{CH}_{3} \mathrm{OH}$ (9:1 vol: vol) solutions and BNZ at $5 / 100(\mathrm{~mol} / \mathrm{mol})$ drug to lipid ratio. Two different $\mathrm{BNZ}$ solvents were used: DMSO and $\mathrm{Cl}_{3} \mathrm{CH}: \mathrm{CH}_{3} \mathrm{OH}: \mathrm{H}_{2} \mathrm{O}$ 1:1:0.3 vol: vol: vol (CMW), both at $60 \mu \mathrm{mol} \mathrm{BNZ} \mathrm{ml}{ }^{-1}$. The lipid-BNZ mixtures were rotary evaporated at $50{ }^{\circ} \mathrm{C}$ in round bottom flasks until the organic solvent was eliminated. The resulting films were flushed with $\mathrm{N}_{2}$, and suspended in $500 \mu \mathrm{l}$ of 10 $\mathrm{mM}$ Tris $-\mathrm{HCl}$ buffer ( $\mathrm{pH}$ 7.4) up to a final concentration of around $20-30 \mu \mathrm{mol} \mathrm{m}{ }^{-1}$ total lipids. The lipid concentrations were determined 
by a colorimetric phosphate micro assay (Bötcher et al., 1961).

\subsection{Separation of unentrapped $B N Z$ from liposomes}

The liposomes were centrifuged in a microcentrifuge EBA12 Hettich Zentrifugen according to the following protocol: (a) $500 \mu \mathrm{l}$ of $\mathrm{BNZ}-$ liposomal suspensions (L-BNZ) were centrifuged at $14000 \mathrm{rpm} 10 \mathrm{~min}$. (b) The upper aqueous phase was removed and discarded; the liposome pellet (volume of $75 \pm 5 \mu$ l) was resuspended in 500 $\mu \mathrm{l}$ of fresh buffer. (c) The process was twice repeated, at an interval of 10 min between each other.

\subsection{Determination of drug/total lipid ratio (D/TL) ( $g$ BNZ/100 g total lipids)}

The $D / \mathrm{TL}$ ratio expressed as $\mathrm{g} \mathrm{BNZ} / 100 \mathrm{~g}$ Total Lipids was determined after complete disruption of one volume of liposomal suspension, in 10 volumes of DMSO. The lipid amounts corresponded to different matrices after three steps of pelleting and buffer replacement. The amount of loaded drug was measured by reverse phase HPLC (Walton and Workman, 1986). HPLC was performed on a Beckman System Gold with a Programmable Solvent Module 125 and a Programmable Detector Module 166. The C18 reverse phase column was Kromasil 100, $5 \mu \mathrm{m}, 25 \times 0.4$ $\mathrm{cm}$ id. The mobile phase used was acetonitrile/ water $(35 / 65)$, with a flow rate of $0.9 \mathrm{ml} \mathrm{min}^{-1}$; sample injection volume was $20 \mu$ detected at 324 nm. A calibration curve for BNZ in DMSO at concentrations of $520,345,260,173,130,86.5,18$, 7, 3.5, 1.7 and $0.35 \mu \mathrm{g} \mathrm{ml}^{-1}$ were prepared. Peak areas were automatically calculated by the equipment; the assay was validated using calibration solutions of $\mathrm{BNZ}$ in absence and presence of HSPC, eggPC and Chol. The results showed no differences between BNZ chomatograms at both conditions. A typical BNZ chromatogram is shown in Fig. 1.
2.6. In vitro assays to predict in vivo performance of formulations

\subsubsection{Release of drug upon dilution assays}

The percentage of drug retention in liposomal structures after dilution was determined according to Amselem (Amselem et al., 1993). Briefly, $10 \mu \mathrm{l}$ of liposomal suspensions were diluted 450 -folds in $10 \mathrm{mM}$ buffer Tris- $\mathrm{HCl}$ (pH 7.4) up to a final volume of $4.5 \mathrm{ml}$ and incubated at $37{ }^{\circ} \mathrm{C}$. After the incubation $(1,10,20$ and $60 \mathrm{~min})$ the tubes were centrifuged for $10 \mathrm{~min}$ at $14000 \mathrm{rpm}$. The $D /$ TL ratios were measured for each liposome pellet, as described in Section 2.5.

\subsubsection{Extent of opsonization}

The amount of plasma proteins associated to the different formulations was measured by a flotation technique in Nycodenz, according to Ford and Rickwood (Ford and Rickwood, 1982; Rickwood et al., 1982; Kelley and Kruski, 1986). Briefly, 100 $\mu 1$ liposomal suspensions and $250 \mu \mathrm{l}$ of human plasma were incubated for $1 \mathrm{~h}$ at $37{ }^{\circ} \mathrm{C}$. Then, the mixtures were diluted in a $13.8 \% \mathrm{w} / \mathrm{v}$ isotonic solution of Nycodenz in A Buffer at 1:1 vol:vol [A Buffer: 1 vol $0.75 \% \mathrm{NaCl}, 1$ vol solution $0.3 \mathrm{mM}$ $\mathrm{CaNa}_{2}$ EDTA, $3 \mathrm{mM} \mathrm{KCl}, 5 \mathrm{mM}$ Tris- $\mathrm{HCl} \mathrm{pH}$ 7.5] (291 mOsm), to a final volume of $4 \mathrm{ml}$ in Ultra-Clear tubes, and completed on the top with saline buffer. The tubes were ultracentrifuged at $150000 \times g$ for $1 \mathrm{~h}$ (SW40 rotors). After centrifugation, liposomes were collected as a milky interface between the Nycodenz and the upper aqueous phase. Liposomal lipids were determined after extraction (Wessel and Flugge, 1984) and their associated plasma proteins were determined by a modified Bradfford microassay (Bradford, 1976).

\section{Results and discussion}

\subsection{BNZ partitioning in phospholipid membranes and choice of liposomal carrier}

In this study, we determined $\mathrm{Kp}$ by a simple procedure which does not require the separation of free drug from membranes. The approach is an equilibrium method based on the concentration- 


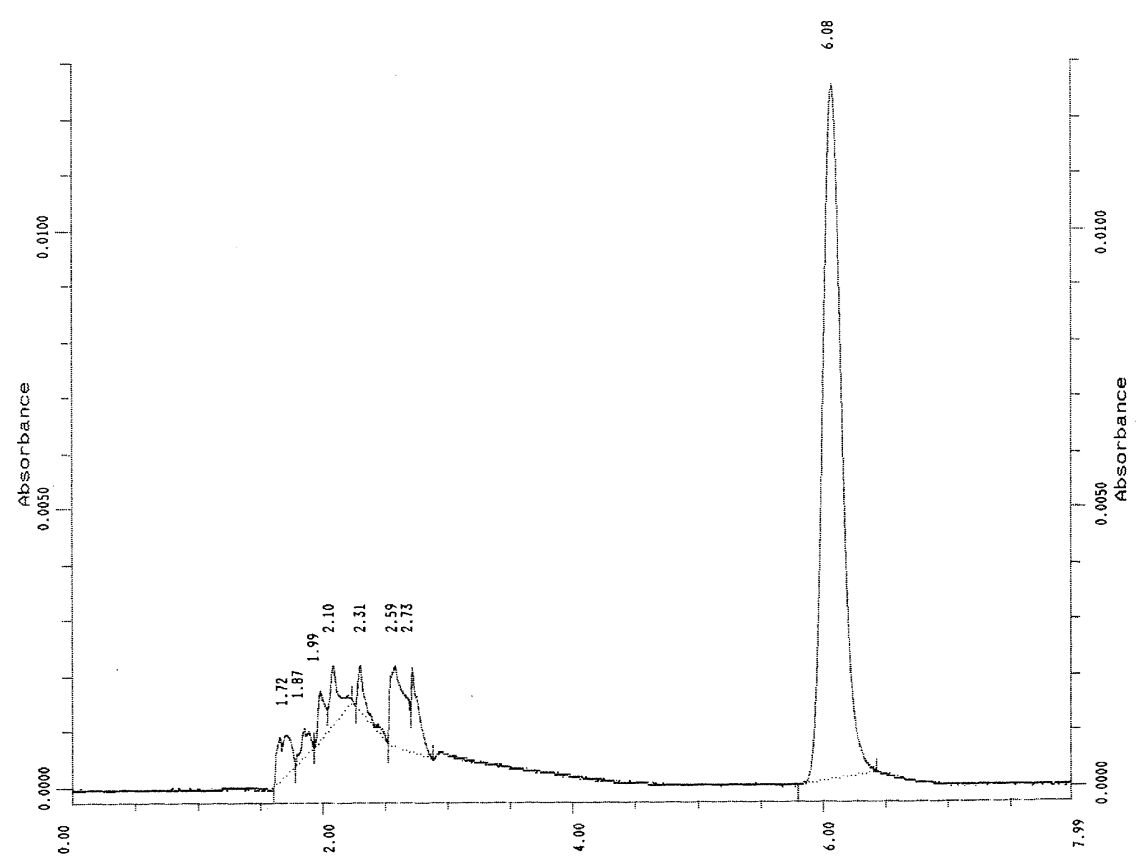

Fig. 1. Chromatogram of BNZ $\left(7 \mu \mathrm{g} \mathrm{ml}^{-1}\right)$ in DMSO.

dependent effect of a partitioning drug on fluorescence of a membrane-incorporated probe. We have chosen the probe Laurdan, a molecule possessing a fluorescent moiety of extreme sensitivity to the polarity of the environment. Laurdan locates at the hydrophobic-hydrophilic interface of the lipid bilayer. It anchors to the membrane by a 12-carbon lauric acid tail at the phospholipid acyl chain region, and its fluorescent naphthalene residue locates close to the glycerol backbone (Parasassi et al., 1991). The Laurdan spectral properties are based on the capacity of sensing polarity and molecular dynamics of dipoles in their own environment, due to dipole relaxation processes. In the liquid crystalline phase of phospholipid bilayers, Laurdan emission displays a maximum at about $490 \mathrm{~nm}$; its emission spectrum is blue shifted in non-polar solvents (Parasassi et al., 1990). In the absence of membranes, Laurdan emission spectrum in methanol decreased upon the addition of BNZ (data not shown). We considered this decrease in Laurdan fluorescence intensity, resulting from the interaction between $\mathrm{BNZ}$ and liposomal membranes, as product of a quenching phenomena caused by BNZ on the fluorophore structure.

Fig. 2a shows the effect of BNZ addition upon the normalized fluorescence intensity: $\left(I_{0} / I\right)$, where $I_{0}$ is the emission intensity (at $\lambda \max =490 \mathrm{~nm}$ ) in absence of BNZ and $I$ is the emission intensity at the same wavelength, in presence of BNZ. Sample's absorbance at $\lambda=360$ (the $\lambda$ max absorption intensity) and $\lambda=490 \mathrm{~nm}$ were lower than 0.5 , therefore, it was unnecessary to correct the signals due to the internal filter effect. The intensity of the emission spectra was unaffected by the presence of methanol, when added at equals volumes than used for adding BNZ.

Each line plotted corresponded to a fixed membrane volume. The horizontal dashed line linked the points of same BNZ concentration in membranes.

The BNZ hydrophobic/hydrophilic balance expressed as $\mathrm{Kp}$ was derived from the following relations (Lissi et al., 1990): $\mathrm{Kp}=\left[n_{\mathrm{M}} / V_{\mathrm{M}}\right] /\left[n_{\mathrm{H}_{2} \mathrm{O}} /\right.$ $\left.V_{\mathrm{H}_{2} \mathrm{O}}\right] \Rightarrow \mathrm{Kp}=C_{\mathrm{M}} /\left[\left(n_{\mathrm{T}}-n_{\mathrm{M}}\right) / V_{\mathrm{H}_{2} \mathrm{O}}\right] \Rightarrow n_{\mathrm{T}}=$ $C_{\mathrm{M}} V_{\mathrm{H}_{2} \mathrm{O}} / \mathrm{Kp}+\mathrm{C}_{\mathrm{M}} V_{\mathrm{M}}$. Kp was obtained from the slope of a graph of $n_{\mathrm{T}}$ versus $V_{\mathrm{M}}$, where $\mathrm{Kp}=$ 


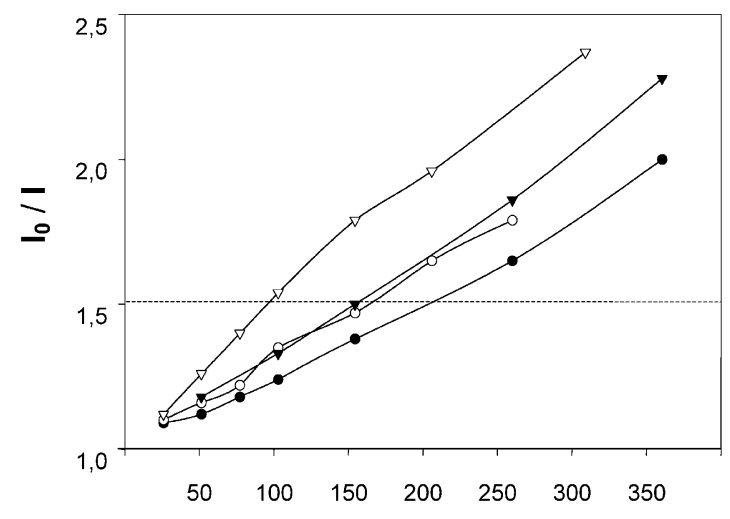

(a) nmol BNZ added to the LUVETs suspension

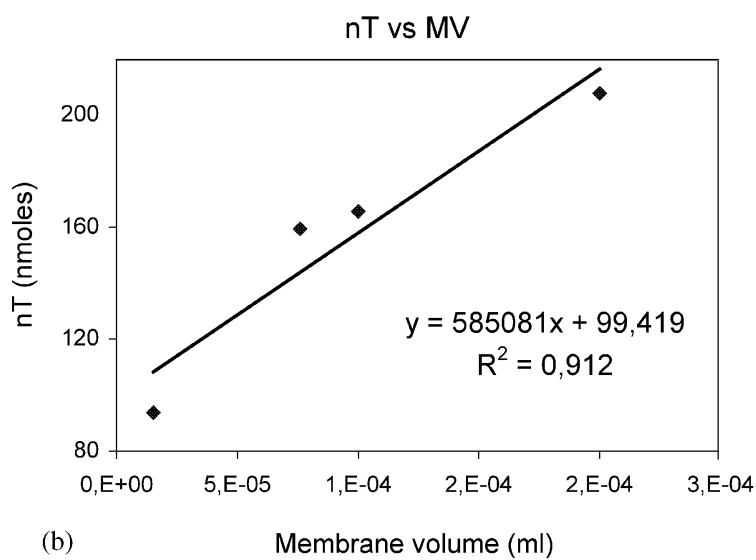

Fig. 2. (a) Effect of increased amounts of BNZ on Laurdan in Large Unilamellar Vesicles obtained by extrusion (LUVETs) fluorescence. The effect was measured at four different liposomal concentrations: $(\bigcirc) 0.15 \mathrm{mM},(\bigcirc) 0.10 \mathrm{mM},(\boldsymbol{\Delta}) 0.05 \mathrm{mM}$ and $(\Delta) 0.01 \mathrm{mM}$. BNZ was added up to the following total lipid:BNZ molar ratios: (1:0.1), (1:0.2), (1:0.3), (1:0.4), (1:0.6), (1:0.9), (1:1.8), (1:3.6) and (1:6.7). (b) BNZ's hydophobic/ hydrophilic balance.

slope. $V_{\mathrm{H}_{2} \mathrm{O}}$ /intersection, as shown in Fig. $2 \mathrm{~b}$. According to this, the calculated $\mathrm{Kp}$ for $\mathrm{BNZ}$ in $\mathrm{PC}$ liposomes was $\cong 10^{4}$.

\subsection{Rationale of drug and lipid starting amounts}

Based on the partitioning behaviour of BNZ in phospholipid membranes, multilamellar vesicles (MLV) were chosen as the carrier system. Such liposomes contain multiple lamellae capable of loading higher mass of hydrophobic drug than unilamellar vesicles. To prepare the multilamellar liposomes, lipids and BNZ were co-solubilised, and MLV were prepared by a thin film method (Bangham and Horne, 1964; Bangham et al., 1965). Because of its simplicity, this proved to be the best method to incorporate hydrophobic drugs into phospholipid membranes (Lasic, 1993a).

The usual working concentration of liposomal suspensions ranges between 10 and $100 \mathrm{mM}$ total lipids (Lasic, 1993b). To load BNZ in MLV, and to remove the non encapsulated drug by centrifugation, the drugs's solubility in aqueous buffer should be the limiting factor of BNZ mass added to the lipids. Drug concentrations above BNZ solubility present crystals in the buffer, which are hard to remove by centrifugation (Batavia et al., 2001). The use of a total drug concentration (total drug $=$ free drug + encapsulated drug) less than its solubility in aqueous buffer $\left(0.40 \mathrm{mg} \mathrm{ml}^{-1}\right)$ (The Merck Index, 2002) ensured that, even if untrapped in liposomes, the drug will remain in solution making its removal by centrifugation possible. Because of this, for a total lipid concentration of $20-30 \mathrm{mM}$, we decided not to increase the BNZ/Total Lipid (TL) ratio beyond the ratio of $5 \mathrm{~mol}$ of BNZ/100 mol TL. Note that the lipid concentration was kept low, while the drug mass was as high as possible. This is consistent with the idea of designing drug carriers with a high payload, and a minimum lipid mass (Lasic, 1993a).

\subsection{D/TL ratio after three steps of pelleting and buffer replacement}

In the electron micrographs from Fig. 3a and b, the retention of sealed vesicular structures after the three steps of pelleting and buffer replacement, in the absence and in the presence of BNZ is shown.

In Fig. 4a, depending on the solvent for BNZ, a difference between the BNZ payload was obtained despite starting from the same $D / T L$ ratio and same lipid matrices. Clearly, vesicles prepared from BNZ dissolved in DMSO loaded between 5 and 10 -fold more drug than those prepared with BNZ dissolved in CMW. The Fig. 4b illustrates the $D /$ TL ratio as a function of lipid matrix, for 

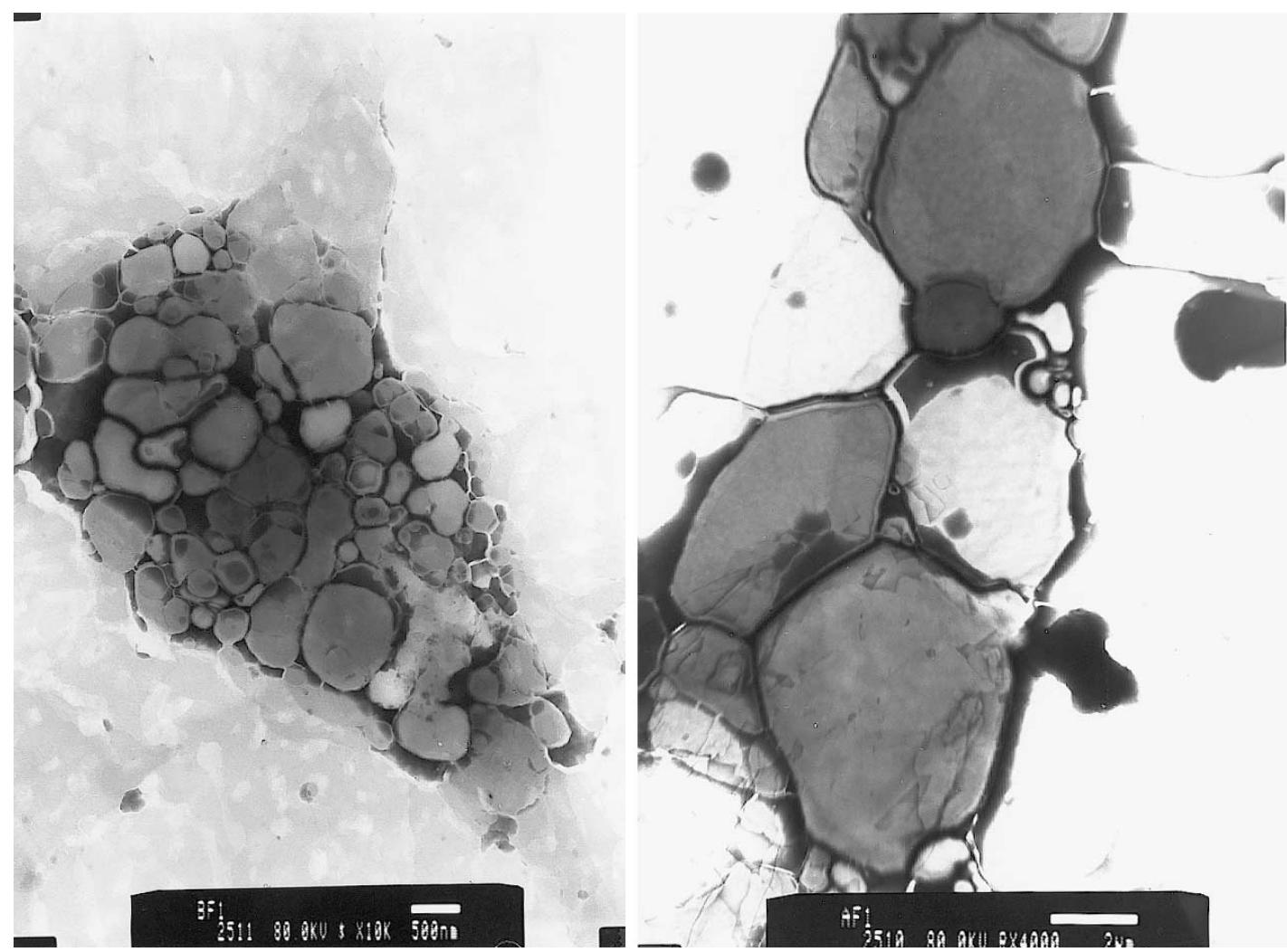

Fig. 3. (a) and (b) electron micrographs of multillamelar vesicles without or with BNZ, respectively. Liposomes were negatively stained with tungsten molibdate.

liposomes prepared with BNZ dissolved in DMSO. The results can be divided in three groups: (a) neutral (physiological $\mathrm{pH}$ ) pure or binary mixtures (eggPC), (HSPC), (HSPC:DPPE 4:1 mol:mol), (eggPC:DPPE 4:1 mol:mol), (eggPC:Chol 1:1 mol:mol), (HSPC:Chol 1:1 mol:mol); (b) neutral (physiological $\mathrm{pH}$ ) ternary mixtures (HSPC:Chol:DPPE 2:2:1 mol:mol:mol), (eggPC:Chol:DPPE 2:2:1 mol:mol:mol) and (c) negatively charged binary or ternary mixtures (eggPC:DSPG 4:1 mol: mol), (HSPC:DSPG1:1 mol:mol), (eggPC:Chol:DSPG 2:2:1 mol:mol:mol), (HSPC:Chol:DSPG 2:2:1 mol:mol:mol). Regarding to the a group, our results pointed to a general requirement of a rigid matrix in order to favour the BNZ incorporation into membranes. The rigid HSPC matrix (phase transition temperature $(\mathrm{Tc})$ for HSPC: $51{ }^{\circ} \mathrm{C}$ ) bilayers incorporated 4-fold more $\mathrm{BNZ}$ than eggPC membranes. It is known that the presence of Chol decreases the fluidity of fluid membranes whereas it increases the fluidity of the rigid ones, until no transition of phases occurs (Chapman, 1975; Almeida et al., 1992). In our systems, Chol reduced the amount of BNZ incorporated in rigid HSPC-matrices but increased it in fluid eggPC matrices, pointing again to the preference of BNZ molecules for a rigid matrix. Following with the $b$ group, ternary mixtures of lipids showed to be a favourable matrix for BNZ incorporation. Concluding with the c type samples, we observed that both in fluid (eggPC) binary matrix, or in rigid (HSPC) binary matrix, the DSPG addition enhanced the BNZ load. Defects and intrinsic instabilities can promote the BNZ inclusion in the lipid bilayer because such discontinuities can accommodate small drug molecules without inducing an additional general disorder that would be energetically unfavourable. These 

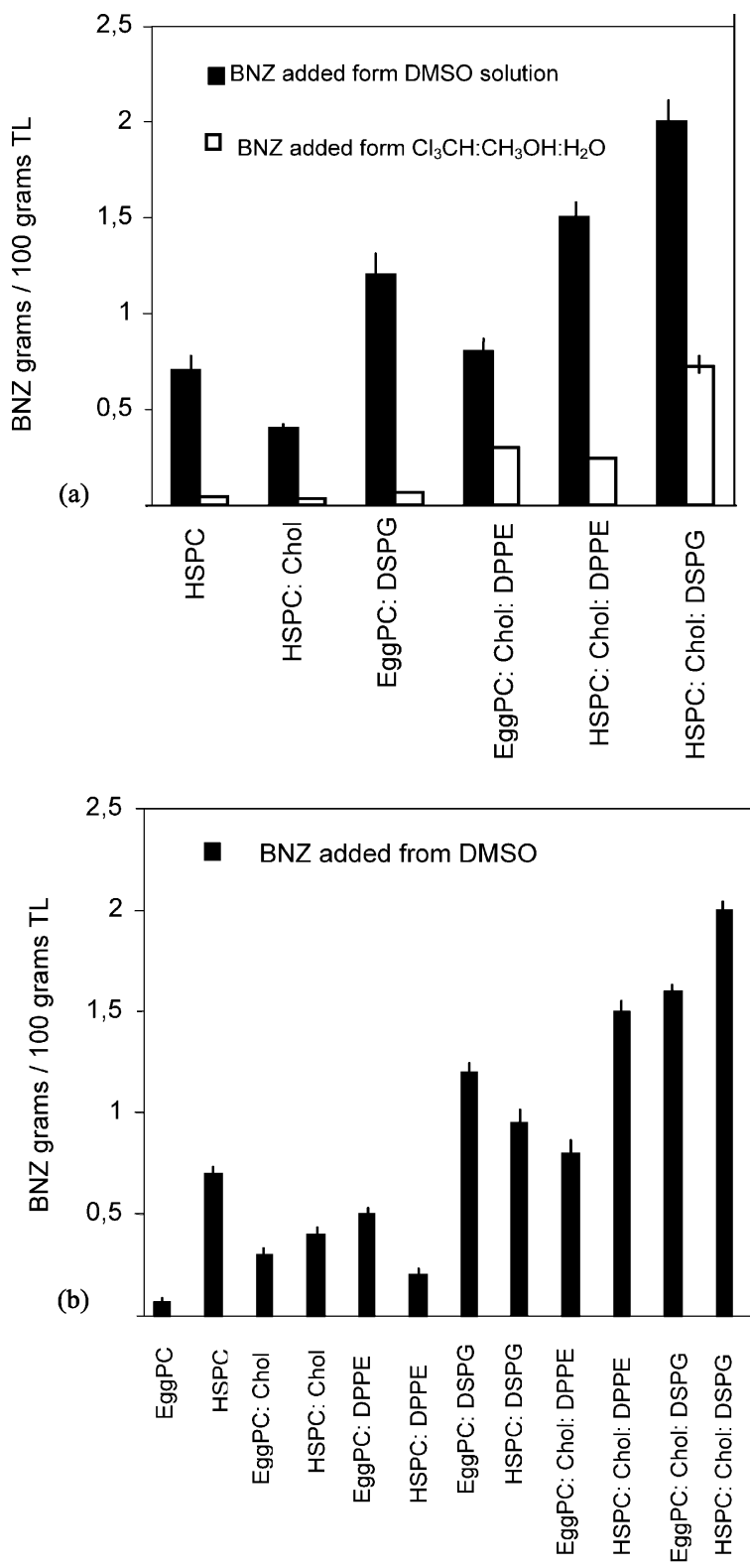

Fig. 4. (a) $D /$ TL ratio corresponding to formulations prepared from BNZ dissolved in DMSO or in $\mathrm{Cl}_{3} \mathrm{CH}: \mathrm{CH}_{3} \mathrm{OH}: \mathrm{H}_{2} \mathrm{O}$ 1:1:0.3 v:v:v (CMW). (b) D/TL ratio as function of lipid composition for liposomes prepared from BNZ in DMSO. The mean lipid recovery for the liposome pellets was $80-90 \%$ in all cases, independently of the presence of BNZ.

local defects may arise either from lipid mixtures (Mui et al., 1994) or from the structural defects known to exist in liposomes below the phase transition temperature (Lee, 1977). Probably a combination of separated lipid domains and the presence of bulky and negatively charged polar head, should favour the partition of BNZ into the boundary lipid domains, as seen in mixtures of $b$ and $\mathrm{c}$ groups. According to our results, the optimal combination for an efficient capture of BNZ in liposomes was a ternary mixture of Chol, the rigid phospholipid HSPC and the negatively charged bulky headgroup DSPG in molar ratio 2:2:1. By employing HSPC: Chol: DSPG as lipid matrix, and dissolving BNZ in DMSO, we ob-

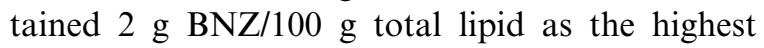
percentage of loaded drug.

\subsection{Dilution assays}

The next step was to perform an in vitro assay, in order to predict how strongly the loaded drug was retained in membranes after liposomal dilution. Fig. 5 shows the percentages of drug retention in liposomes when diluted 450-fold in Tris buffer, as a function of time. Table 1 shows the rate of drug release, divided between the first and the next $59 \mathrm{~min}$, together with the $D /$ TL ratio after $60 \mathrm{~min}$ of dilution. Independently of lipid composition, there was fast drug lost at the beginning, followed by a second step where most formulations stopped leaking and a few continued losing drug at different rates. The existence of an initial fast drug loss from membranes, followed by multiple slower steps of drug loss is characteristic of liposomal multilamellar formulations, for instance doxorubicin (L-DXR) (Amselem et al., 1993), in which the initial rate is commonly ascribed to drug detachment from liposomal surface. The following period should be the product of impaired release of drug from the inner lamellae (Amselem et al., 1993).

In our results, the two rigid formulations (HSPC: Chol and HSPC: Chol: DSPG) exhibited an exceptional behaviour, with a slower rate in the first minute (45\% (drug lost) $\mathrm{min}^{-1}$ and $65 \%$ (drug lost) $\mathrm{min}^{-1}$ ), followed by a rate of $0.5 \%$ (drug lost) $\min ^{-1}$ and $0.4 \%$ (drug lost) $\min ^{-1}$ for the next 59 min. Remarkably, the HSPC: Chol: DSPG formulations presented a $D / \mathrm{TL}$ ratio $2-25$-fold higher than the corresponding ratio for other 

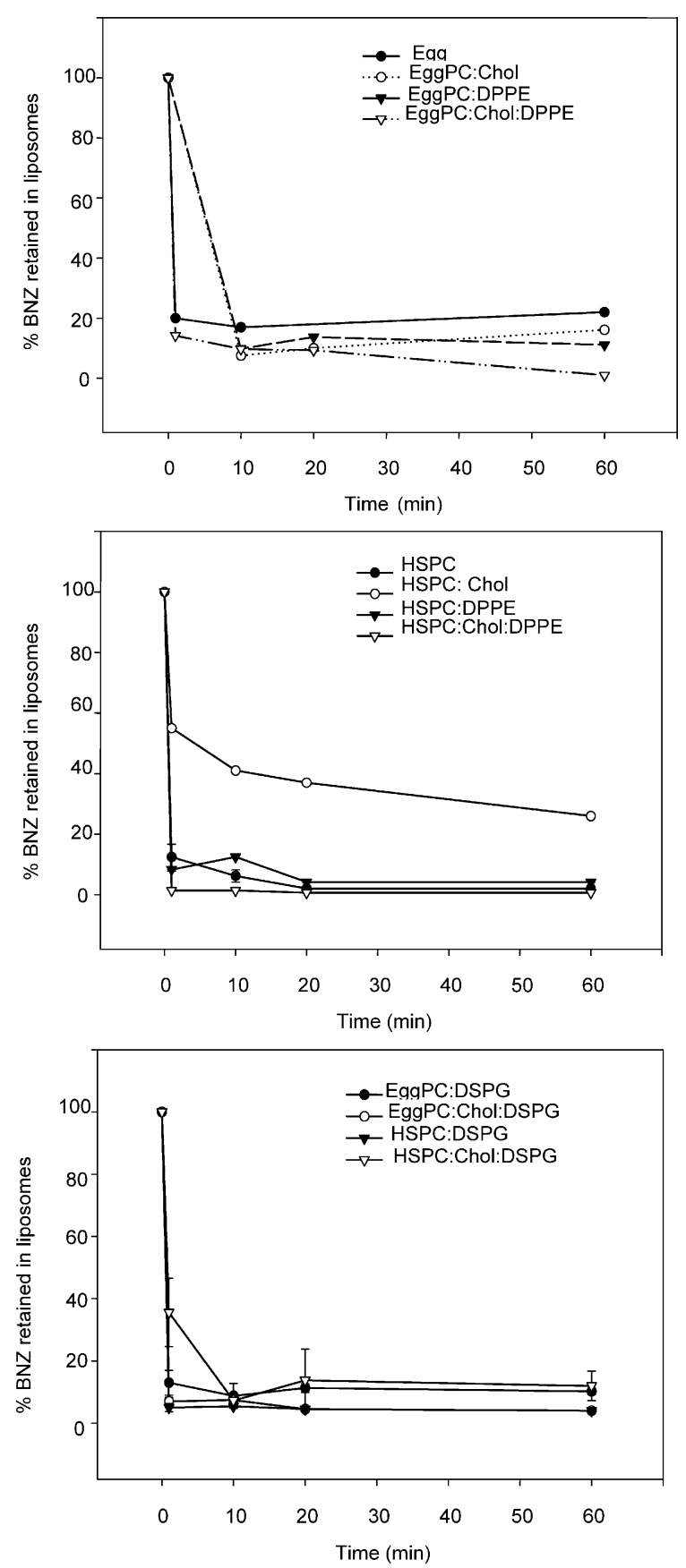

Fig. 5. Dilution assay in Tris buffer. The drug/lipid ratios were determined at $0,1,10,20$ and $60 \mathrm{~min}$, as described in Section 2.6.1. formulations. Thus, we selected HSPC: Chol: DSPG as the best lipid matrix, because it combined the highest amount of loaded BNZ before being diluted, together with the highest drug retention after dilution.

\subsection{Protein binding assay}

The second in vitro assay was the measurement of the level of protein adsorption on BNZ loaded and empty liposomes. From the difference between empty and loaded vesicles, an estimation of the in vivo effect of $\mathrm{BNZ}$ on the level of protein binding to membranes and hence, their probable physical stability, was obtained.

The ratio $r=\mu \mathrm{g}$ of protein adsorbed to liposomes/ $\mu \mathrm{mol}$ of liposomal lipids is a direct estimation of $k c$, the rate of particle clearance from circulation. As stated before, the role of adsorbed proteins on the liposomal surface is extremely important because the two main contributions to $k c$ (liposomal physical integrity and rate of macrophage uptake) depend on it (Semple et al., 1996). Therefore, the in vitro estimation of $r$ is a useful guideline to predict the in vivo performance of liposomal formulations.

Our results, depicted in Fig. 6, indicated that $r$ for liposomes containing BNZ was substantially higher than the $r$ for empty vesicles. Particularly, BNZ rigid HSPC matrices adsorbed 2-24-fold more proteins than BNZ-fluid eggPC matrices. The existence of this effect in rigid liposomes was independent of the Chol presence. BNZ-fluid egg$\mathrm{PC}$ liposomes presented a lower $\mathrm{r}$ than rigid ones, but the inclusion of a negative charge (DSPG) increased $\mathrm{r}$ 3-16-fold as compared with $r$ for neutral fluid liposomes.

The principal factors responsible for the lifetime in circulation of a liposomal particle are two: (a) the rate of liposomal metabolism: rigid vesicles are slowly processed by the RES cells, inducing a temporary impairment on general particle phagocitosis (Kao and Juliano, 1981; Harashima et al., 1992) and (b) the level of plasma protein adsorption on membranes. Plasma protein adsorption is proportional to the exposition of hydrophobic defects, and decreases with the inclusion of Chol (Abra and Hunt, 1981; Semple et al., 1996). 
Table 1

Rate of drug release for the different liposomal composition and drug retention in liposomes after dilution

\begin{tabular}{llll}
\hline Composition & $\begin{array}{l}\text { Rate of drug release in } \\
\text { the first minute }\end{array}$ & $\begin{array}{l}\text { Rate of drug release for the } \\
\text { following } 59 \mathrm{~min}^{\mathrm{a}}\end{array}$ & $\begin{array}{l}\text { Drug retention in liposomes (g BNZ/100 g total } \\
\text { lipids) after 60 min at 450-fold dilution }\end{array}$ \\
\hline EggPC & 80 & $\cong 0$ & 0.09 \\
EggPC:Chol & 90 & $\cong 0$ & 0.07 \\
EggPC:DPPE & 90 & $\cong 0$ & 0.06 \\
EggPC:Chol:DPPE & 85 & 0.2 & 0.01 \\
HSPC & 87 & 0.2 & 0.01 \\
HSPC:Chol & 45 & 0.5 & 0.07 \\
HSPC:DPPE & 90 & 0.1 & 0.01 \\
HSPC:Chol:DPPE & 99 & $\cong 0$ & 0.01 \\
EggPC:DSPG & 85 & 0.1 & 0.12 \\
EggPC:Chol:DSPG & 93 & 0.06 & 0.12 \\
HSPC:DSPG & 95 & 0.03 & - \\
HSPC:Chol:DSPG & 65 & 0.4 & 0.25 \\
\hline
\end{tabular}

a The rate of drug release is express as $\%$ drug lost per minute.

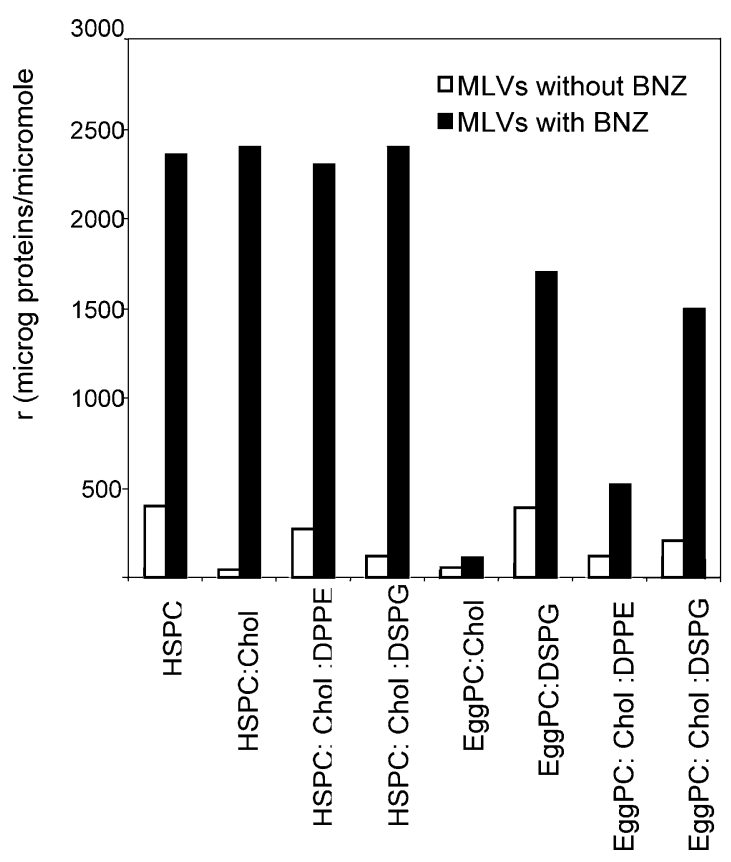

Fig. 6. Level of protein adsorption, measured as $r$ ( $\mu$ g proteins/ $\mu \mathrm{mol}$ TL).

Plasma protein adsorption also depends on the lipid charge; negatively charged lipids (except phosphatidyl inositol or GM1) usually bind larger amount of proteins than positively or neutral lipids of the same size (Scherphof et al., 1978; Hwang, 1987).
Overall, drugs presenting a high Kp ( $>1000)$ (Lasic, 1993a) can be successfully loaded in liposomes. Our results rendered a $\mathrm{Kp}$ in the order of $10^{4}$; therefore, we considered the loading of MLV with BNZ as feasible. In the next step we generated a set of different lipid matrices combined with BNZ dissolved in different organic solvents; from the obtained MLVs, the formulation with the highest $D /$ TL ratio was selected. It is useful to note that the drug's solubility in water is not necessarily inversely related to $\mathrm{Kp}$, which indicates a drug's maximal concentration at a given temperature. For example, Doxorubicin's solubility in water is high; however, its $\mathrm{Kp}$ is high enough to be loaded in liposomes of eggPC: eggPG: Chol 7:3:4 at a $5 \mathrm{~g}$ DXR/100 g TL (Amselem et al., 1993). On the contrary, BNZ's solubility is low: $0.4 \mathrm{mg} \mathrm{ml}^{-1}$ at $\mathrm{pH} 7$, and there is concern about the physical stability of carriers loaded with poorly soluble drugs: when liposomes are strongly diluted, hydrophobic drugs tend to escape from the liposomal matrix toward the bulk medium. If dilution is produced in plasma, a sink effect is added by the presence of plasma proteins (Scheider, 1985; Amselem et al., 1993). Consequently, extra amounts of drug are lost from the carrier. Because of this we investigated in vitro the future performance of BNZ-HSPC:DSPG:Chol as pharmacological carriers. The dilution assays showed high and biphasic rates of $\mathrm{BNZ}$ release, and for BNZ- 
HSPC liposomes, a level of protein adsorption (proportional to in vivo $k c$ ) that was unusually high. In view of these results, some instability when diluted is expected for this formulation, but probably a fast capture by RES cells will result.

\section{Conclusions}

The Kupffer cells (KC) in the liver and the spleen macrophages are major natural targets for multilamellar liposomes. Therefore, we consider BNZ loaded multilamellar liposomes as potentially useful to eradicate the amastigote nests in the cytoplasm of KC. The employment of liposomal formulation of $\mathrm{BNZ}$ instead of free $\mathrm{BNZ}$ should increase the amount of drug delivered to infected $\mathrm{KC}$.

\section{Acknowledgements}

This research was supported by a grant from the Universidad Nacional de Quilmes. Maria J. Morilla has got a post-graduate fellowship from the Comisión de Investigaciones Científicas (CIC) de Buenos Aires, Argentina.

\section{References}

Abra, R.M., Hunt, C.A., 1981. Liposome distribution in vivo: III dose and vesicle size effect. Biochim. Biophys. Acta 666, 493-503.

Almeida, P.F.F., Vaz, W.L.C., Thomson, T.E., 1992. Lateral diffusion in the liquid phases of dimyristoylphosphatidylcholine/cholesterol lipid bilayers: a free volume analysis. Biochemistry 31, 6739-6747.

Amselem, S., Cohen, R., Barenholz, Y., 1993. In vitro tests to predict in vivo performance of liposomal dosage forms. Chem. Phys. Lipids 64, 219-237.

Bangham, D., Horne, R.W., 1964. Negative staining of phospholipids and their structural modifications by surface active agents as observed in the electron microscope. J. Mol. Biol. 8, 660-668.

Bangham, D., Standish, M.M., Watkins, J.C., 1965. Diffusion on univalent ions across lamellae of swollen phospholipids. J. Mol. Biol. 13, 238-252.

Batavia, R., Taylor, K.M.G., Craig, D.Q.M., Thomas, M., 2001. The measurement of beclomethasone dipropionate entrapment in liposomes: a comparison of a microscope and an HPLC method. Int. J. Pharm. 212, 109-119.

Bötcher, C.J.F., van Gent, C.M., Pries, C., 1961. A rapid and sensitive sub-micro phosphorus determination. Anal. Chim. Acta 24, 203-204.

Bradford, M.M., 1976. A rapid and sensitive method for the quantitation of microgram quantities of protein utilising the principle of protein-dye binding. Anal. Biochem. 86, $142-$ 146.

Chapman, D., 1975. Phase-transitions and fluidity characteristics of lipids and cell membranes. Q. Rev. Biophys. 8, 185235.

Coura, J.R., 1996. Current prospects of specific treatment of Chagas' disease. Bol. Chil. Parasitol. 51, 69-75.

Ford, T., Rickwood, D., 1982. Formation of isotonic nycodenz gradients for cell separations. Anal. Biochem. 123, 293-298.

Harashima, H., Kume, Y., Kiwada, H., 1992. Non-MichaelisMenten type hepatic uptake of liposomes in the rat. J. Pharm. Pharmacol. 44, 707-712.

Hwang, K.J., 1987. Liposome pharmacokinectis. In: Ostro, M.J. (Ed.), Liposomes: from Biophysics to Therapeutics. Marcel Dekker, New York, pp. 109-156.

Kao, Y.J., Juliano, R.L., 1981. Interactions of liposomes with the reticuloendothelial system. Effect of reticuloendothelial blockade on the clearance of large unilamellar vesicles. Biochim. Biophys. Acta 677, 453-461.

Kelley, T.L., Kruski, A.W., 1986. Density gradient ultracentrifugation of serum lipoproteins in a swinging bucket rotor. Methods Enzymol. 128, 170-181.

Lasic, D.D., 1993. Liposomes for drug delivery system. In: Lasic, D.D. (Ed.), Liposomes from Physics to Applications (Chapter 11). Elsevier Science BV, pp. 265-321.

Lasic, D.D., 1993. General Introduction to liposomes. Preparation of liposomes. In: Lasic, D.D. (Ed.), Liposomes from Physics to Applications. Elsevier, pp. 63-108.

Lee, A.G., 1977. Analysis of the defect structure of gel-phase lipid. Biochemistry 5, 835-841.

Lissi, E., Bianconi, M.L., do Amaral, A.T., De Paula, E., Blanch, L.E., Schreirer, R., 1990. Methods for the determination of partition coefficients based on the effects of solutes upon membrane structure. Biochim. Biophys. Acta $1021,45-50$.

Mui, B.L., Cullis, P.R., Pritchard, P.H., Madden, T.D., 1994. Influence of plasma on the osmotic sensitivity of large unilamellar vesicles prepared by extrusion. J. Biol. Chem. 269, 7360-7364.

Parasassi, T., de Stasio, G., d'Ubaldo, A., Gratton, E., 1990. Phase fluctuation in phospholipid membranes revealed by Laurdan fluorescence. Biophys. J. 57, 1179-1186.

Parasassi, T., de Stasio, G., Ravagnan, G., Rusch, R.M., Gratton, E., 1991. Quantitation of lipid phases in phospholipid vesicles by GP of Laurdan fluorescence. Biophys. J. 60, 179-189.

Rickwood, D., Ford, T., Graham, J., 1982. Nycodenz: a new non-ionic iodinated gradient medium. Anal. Biochem. 56, $23-31$. 
Scheider, M., 1985. Liposomes as drug carriers: 10 years of research. In: Buri, P., Gumma, A. (Eds.), Drug Targeting. Elselvier, New York, NY, pp. 119-134.

Scherphof, G., Roerdink, F., Waite, M., Parks, J., 1978. Disintegration of phosphatidilcholine liposomes in plasma as a result of interaction with high density lipoproteins. Biochim. Biophys. Acta 842, 296-307.

Semple, S.C., Chonn, A., Cullis, P.R., 1996. Influence of cholesterol on the association of plasma proteins with liposomes. Biochemistry 35, 2521-2525.

The Merck Index, 2002. An encyclopaedia of chemicals, drugs and biologicals, Centennial edition, Budavari, S. (Ed.), Publish by Merck \& Co, Inc., Rahway, NY, USA.
Van Gompel, A., Vervoort, T., 1997. Chemotherapy of leishmaniasis and trypanosomiasis. Curr. Opin. Infect. Dis. 10, 469-474.

Walton, M.I., Workman, P., 1986. Reversed-phase high performance liquid chromatographic methods for the simultaneous determination of the 2-nitroimidazole benznidazole and its amine metabolite in biological materials. J. Chromatogr. 375, 190-193.

Wessel, D., Flugge, U.I., 1984. Method for the quantitative recovery of proteins in dilute solution in the presence of detergents and lipids. Anal. Biochem. 138, 141-143. 